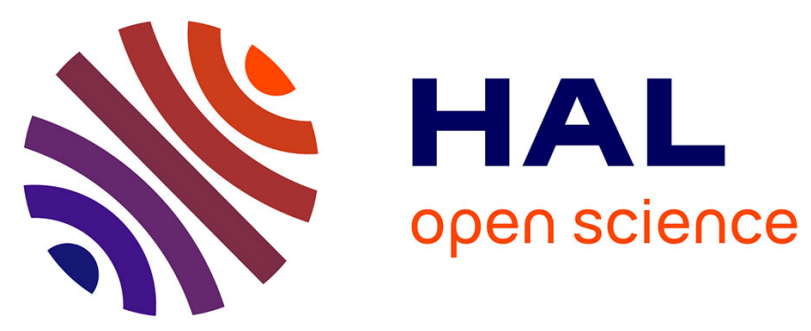

\title{
MULTI-SCALE ANTI-CORRELATION BETWEEN ELECTRON DENSITY AND MAGNETIC FIELD STRENGTH IN THE SOLAR WIND
}

Shuo Yao, J.-S. He, E. Marsch, C.-Y. Tu, A. Pedersen, H. Rème, Jean-Gabriel Trotignon

\section{To cite this version:}

Shuo Yao, J.-S. He, E. Marsch, C.-Y. Tu, A. Pedersen, et al.. MULTI-SCALE ANTI-CORRELATION BETWEEN ELECTRON DENSITY AND MAGNETIC FIELD STRENGTH IN THE SOLAR WIND. The Astrophysical Journal, 2011, 728 (2), pp.146. 10.1088/0004-637X/728/2/146 . insu01310947

\section{HAL Id: insu-01310947 https://hal-insu.archives-ouvertes.fr/insu-01310947}

Submitted on 20 May 2016

HAL is a multi-disciplinary open access archive for the deposit and dissemination of scientific research documents, whether they are published or not. The documents may come from teaching and research institutions in France or abroad, or from public or private research centers.
L'archive ouverte pluridisciplinaire $\mathbf{H A L}$, est destinée au dépôt et à la diffusion de documents scientifiques de niveau recherche, publiés ou non, émanant des établissements d'enseignement et de recherche français ou étrangers, des laboratoires publics ou privés.

\section{(이)(\$)}

Distributed under a Creative Commons Attribution - NonCommercial - NoDerivatives| 4.0 


\title{
MULTI-SCALE ANTI-CORRELATION BETWEEN ELECTRON DENSITY AND MAGNETIC FIELD STRENGTH IN THE SOLAR WIND
}

\author{
Shuo Yao ${ }^{1}$, J.-S. He ${ }^{1}$, E. Marsch ${ }^{2}$, C.-Y. Tu ${ }^{1}$, A. Pedersen ${ }^{3}$, H. Rème ${ }^{4}$, And J. G. Trotignon ${ }^{5}$ \\ ${ }^{1}$ Department of Geophysics, Peking University, Beijing 100871, China; yaoshuo@pku.edu.cn \\ ${ }^{2}$ Max-Planck-Institut für Sonnensystemforschung, Katlenburg-Lindau, Germany \\ ${ }^{3}$ Department of Physics, University of Oslo, Oslo, Norway \\ ${ }^{4}$ Centre d' Etude Spatiale des Rayonnements, Toulouse, France \\ ${ }^{5}$ Laboratoire de Physique et Chimie de I'Environnement et de I'Espace, Orléans, France \\ Received 2010 October 24; accepted 2010 December 10; published 2011 February 1
}

\begin{abstract}
This work focuses on the relation between the electron density and the magnetic field strength in the solar wind, and aims to reveal its compressive nature and to determine the level of compressibility. For this purpose, we choose a period of quiet solar wind data obtained at $1 \mathrm{AU}$ by the Cluster $\mathrm{C} 1$ satellite. The electron density is derived with a sampling time as high as $0.2 \mathrm{~s}$ from the spacecraft-potential measurements made by the Electric Field and Waves instrument. We use the wavelet cross-coherence method to analyze the correlation between the electron density and the magnetic field strength on various scales. We find a dominant anti-correlation between them at different timescales ranging from $1000 \mathrm{~s}$ down to $10 \mathrm{~s}$, a result which has never been reported before. This may indicate the existence of pressure-balanced structures (PBSs) with different sizes in the solar wind. The small (mini) PBSs appear to be embedded in the large PBSs, without affecting the pressure balance between the large structures. Thus, a nesting of these possible multi-scale PBSs is found. Moreover, we find for the first time that the relative fluctuation spectra of both the electron number density and the magnetic field strength look almost the same in the range from $0.01 \mathrm{~Hz}$ to $2.5 \mathrm{~Hz}$, implying a similar cascading for these two types of fluctuations. Probable formation mechanisms for the multi-scale possible PBSs are discussed. The results of our work are believed to be helpful for understanding the compressive nature of solar wind turbulence as well as the connections between the solar wind streams and their coronal sources.
\end{abstract}

Key words: solar wind - turbulence

Online-only material: color figures

\section{INTRODUCTION}

Pressure-balanced structures (PBSs) show a typical anticorrelation between the fluctuations of the thermal pressure $\left(P_{\mathrm{k}}\right)$ and magnetic pressure $\left(P_{\mathrm{B}}\right)$. They have often been observed in the solar wind and are considered to be major ingredients of solar wind turbulence and among the most prominent compressive fluctuations (see a review by Tu \& Marsch 1995 and references therein). The first PBS found in the solar wind was reported by Burlaga \& Ogilvie (1970). They established that $P_{\mathrm{k}}$ and $P_{\mathrm{B}}$ were negatively correlated on the $1 \mathrm{hr}$ scale, indicating the existence of PBSs, but positively correlated on the 2 days scale, resulting from the interaction between high-speed and lowspeed streams. In the ecliptic-plane solar wind, Thieme et al. (1990) found similar evidence for PBSs but of coronal origin, and the correlations between different pairs of parameters, such as density, speed, magnetic field magnitude, and so on, were exhaustively studied by means of the Helios data by Marsch \& Tu (1993) and Tu \& Marsch (1994). A general tendency for an anti-correlation between $P_{\mathrm{k}}$ and $P_{\mathrm{B}}$ was also inferred from data based on both the broadband correlation spectra and the hourly correlation coefficients (Marsch \& Tu 1993). Then, a model invoking a superposition of PBSs and perpendicular fast magnetosonic waves was proposed (Tu \& Marsch 1994) to qualitatively explain the observations of compressive fluctuations in solar wind. In the polar solar wind, the PBSs were also identified and found to exist at scales from half a day down to $1 \mathrm{hr}$ on the basis of Ulysses data (e.g., McComas et al. 1995; Reisenfeld et al. 1999; Bavassano et al.
2004). With Cluster data Kellogg \& Horbury (2005) found possible PBSs with scales of seconds in their work, though they preferred to explain what they observed as ion acoustic wave. However, PBSs with scales from $1 \mathrm{hr}$ to less than 1 minute, which is close to the validity limit of magnetohydrodynamics (MHD), have not yet been revealed together in the same solar wind period. To do that is one main goal of this study.

An anti-correlation between fluctuations of the plasma number density $n$ and magnetic field magnitude $B$ was often observed in association with PBSs (Goldstein \& Siscoe 1972; Tu $\&$ Marsch 1994; Bavassano et al. 2004). The frequency for this $n-B$ anti-correlation studied so far usually ranged from $1 \times 10^{-4}$ to $3 \times 10^{-3} \mathrm{~Hz}$, corresponding to a timescale larger than $5 \mathrm{~min}$ utes. Both the fast and slow streams in the ecliptic solar wind showed the $n-B$ anti-correlation in association with the PBSs (Tu \& Marsch 1994). The role for the $n-B$ anti-correlation relevant to the PBSs was found to become stronger as the solar distance increases and the absolute heliographic latitude decreases (Bavassano et al. 2004). Therefore, in the ecliptic plane at $1 \mathrm{AU}$, the $n-B$ anti-correlation may be used to identify the PBSs.

Rapid measurements of the plasma number density are required to study the $n-B$ anti-correlation at timescales less than 1 minute. Recently, Pedersen et al. (2001, 2008) and Kellogg \& Horbury (2005) reported a method to obtain the electron number density $N_{\mathrm{e}}$ at a high time resolution from the spacecraft-potential measurements by EFW (Electric Field and Waves experiment) on Cluster, using the theory stated in Labelle \& Kintner (1989). Since the spacecraft potential is measured every $0.2 \mathrm{~s}$, this method offers the electron number density sampled at the high 
time resolution of $0.2 \mathrm{~s}$. Given the 0.95 confidence level, PBSs with short timescales on the order of $10 \mathrm{~s}$ can be expected to be revealed from our data.

The fluctuations of $n$ and $B$ are often analyzed with the help of the power spectral density (PSD), when investigating the nature of the solar wind turbulence. In the inertial range above the ion gyro scale, the solar wind turbulence is usually dominated by a cascade of Alfvénic fluctuations and accompanied by a passive cascade of density and magnetic-field-strength fluctuations (Schekochihin et al. 2009). Under the assumption of near incompressibility, it has been demonstrated that the fluctuations of $n$ and $B$ are passively convected by the incompressible velocity fluctuations, and exhibit nearly the same slope $(-5 / 3)$ of PSD (Dastgeer \& Zank 2004). Observationally, the PSDs of $n$ and $B$ fluctuations in the inertial range are found to have a $-5 / 3$ spectral law for the low-speed flows, while they reveal a flattening tail between $5 \times 10^{-4}$ and $2 \times 10^{-3} \mathrm{~Hz}$ for the high-speed flows (Marsch \& Tu 1990). A similar variation of the spectral shape of the $n$ PSD for various solar wind speeds was confirmed again by Kellogg \& Horbury (2005). However, according to our knowledge, the simultaneous PSDs of $n$ and $B$ extending from the inertial range into the dissipation range up to $2.5 \mathrm{~Hz}$ have not yet been given before.

Our research is focused on the relation of the electron density fluctuations and magnetic field fluctuations, trying to reveal the possible PBSs at different scales in the solar wind. The detailed spectra of the $N_{\mathrm{e}}$ and $B$ fluctuations are investigated and compared with the power law predicted for a passive scalar as well as the spectrum resulting from a superposition of active field fluctuations. We use the Morlet wavelet cross-coherence method (Grinsted et al. 2004) to study the cross-correlation between the $N_{\mathrm{e}}$ and $B$ fluctuations at different timescales and different moments in time. Accordingly, the time series obtained for the compressive fluctuations show obvious anti-correlations at different timescales. The probable physical origin of these multi-scale possible PBSs is also discussed.

\section{OBSERVATIONS}

\subsection{Data Processing Method}

At first, we will explain how to derive electron number density $N_{\mathrm{e}}$ with high time resolution from the spacecraft potential. The spacecraft potential is directly measured by the EFW on board the Cluster satellite with a time resolution of 0.2 s (Pedersen et al. 2001, 2008). Among the instruments on Cluster, PEACE (Plasma Electron And Current Experiment) and WHISPER (Waves of High Frequency and Sounder for Probing of Electron Density by Relaxation) could provide the electron number density for each $4 \mathrm{~s}$ and $1.7 \mathrm{~s}$, respectively. In order to improve the time resolution of $N_{\mathrm{e}}$, Pedersen et al. (2008) and Kellogg \& Horbury (2005) suggested a method to obtain the electron number density $\left(N_{\mathrm{e}}\right)$ from the spacecraft potential $\left(V_{\mathrm{sp}}\right)$ measured by the EFW for each $0.2 \mathrm{~s}$. The principle of deriving $N_{\mathrm{e}}$ from $V_{\mathrm{sp}}$ is based on the balance between collected electron current and photoelectron current. The relation between $V_{\text {sp }}$ and $N_{\mathrm{e}}$ can be well fitted by using a linear combination of multiple exponential functions (Kellogg \& Horbury 2005; Pedersen et al. 2008). For our case in practice, we use the $N_{\mathrm{e}}$ data from WHISPER at a lower time resolution and the $V_{\mathrm{sp}}$ data from EFW to establish the fitting function composed of two exponential functions. Based on this fitting function, the $N_{\mathrm{e}}$ with a higher time resolution of $0.2 \mathrm{~s}$ is derived from the $V_{\mathrm{sp}}$ at the same time resolution.
As mentioned in Tjulin et al. (2005), the spin of spacecraft would affect the time series as well as the power spectrum from the EFW measurements. We applied a series of notch filters to remove the effect of the spacecraft spin on the time series of $N_{\mathrm{e}}$. Since we know the spin period of Cluster is $4 \mathrm{~s}$, we set the notch filters at the multiples of $0.25 \mathrm{~Hz}$, from $0.25 \mathrm{~Hz}$ to $2.5 \mathrm{~Hz}$. In this way, no obvious spikes due to the spin effect will appear in the corresponding PSD after notch filtering. In order to decrease the random noise in the PSD, we applied the multitaper method (Percival \& Walden 1993; Eastwood et al. 2009) to depress the random noise.

To study the correlation between $N_{\mathrm{e}}$ and $B$, we introduce the Morlet wavelet cross-coherence method, which was developed by Grinsted et al. (2004). Since we need to discriminate the positive correlation and negative correlation, we show the spectrum of the cross coherence together with the spectrum of the absolute phase angle.

\subsection{Data Analysis}

We choose a period of clean solar wind measured by the Cluster C1 satellite from 22:30 to 23:30 on 2001 April 5, shown in Figure 1. This period has been studied by Alexandrova et al. $(2008,2009)$ to analyze magnetic field spectral in high frequency based on the measurements of Spatio-Temporal Analysis of Field Fluctuations-Search Coils (STAFF-SC). In this period the ion gyro frequency in the plasma frame is $0.11 \mathrm{~Hz}$. The magnetic field strength as measured by Fluxgate Magnetometer (FGM), with $0.2 \mathrm{~s}$ time resolution, stays around $7 \mathrm{nT}$, but the component direction reversals occur several times in this period. The ion number density measured by the Cluster Ion Spectrometry Hot Ion Analyzer (CIS HIA) with small $g$ in solar wind mode (Rème et al. 2001) fluctuated between 2 and $5 \mathrm{~cm}^{-3}$. The time resolution of CIS is $4 \mathrm{~s}$. The perpendicular ion temperature measured by CIS fluctuates around $0.2 \times 10^{6} \mathrm{~K}$. The total pressure in Figure 1 only includes magnetic pressure and ion perpendicular pressure, staying constantly around $0.025 \mathrm{nPa}$. Since we note that the parallel ion temperature is not accurately measured by CIS and the electron temperature measured by PEACE having a data interval up to $160 \mathrm{~s}$ which should normally be $4 \mathrm{~s}$, we did not use them in our study. As a result, we cannot obtain a convincing plasma pressure. Based on the incomplete total pressure, we tend to classify the solar wind period we study as possible pressure balance. The solar wind velocity is around $550 \mathrm{~km} \mathrm{~s}^{-1}$, nearly along the Sun-Earth radial direction.

First, we need to derive $N_{\mathrm{e}}$ with a $0.2 \mathrm{~s}$ time resolution from $V_{\mathrm{sp}}$ (the spacecraft potential is given here in volt and has negative values). The data of spacecraft potential we use are the average value of the four probes on EFW, obtained from the Cluster Active Archive Web site. The fitting function between them for this special period is found to be $N_{\mathrm{e}}\left(\mathrm{cm}^{-3}\right)=$ $22 \exp \left(V_{\mathrm{sp}} / 5.5\right)+0.05 \exp \left(V_{\mathrm{sp}} / 30\right)$ based on the $N_{\mathrm{e}}$ measured by WHISPER. The electron number density calculated from EFW is consistent with that measured by WHISPER, and is shown in the upper panel of Figure 2.

Second, we applied the multitaper and notch filter method (Eastwood et al. 2009) to obtain the corresponding power spectrum of the electron number density from the inertial range to the dissipation range. The Multitaper Fast Fourier Transformation method yields a PSD with small noise level at the higher frequency part. In order to analyze the spectrum as a function of spatial scale in units of the ion gyro radius $\left(\rho_{i}\right)$, we transfer the spectrum from the frequency domain into the $k \rho_{i}$ domain, based on Taylor's hypothesis. We calculate $k=\omega / v_{\mathrm{sw}}$ 
Quiet Solar Wind Period Measured by Cluster-1

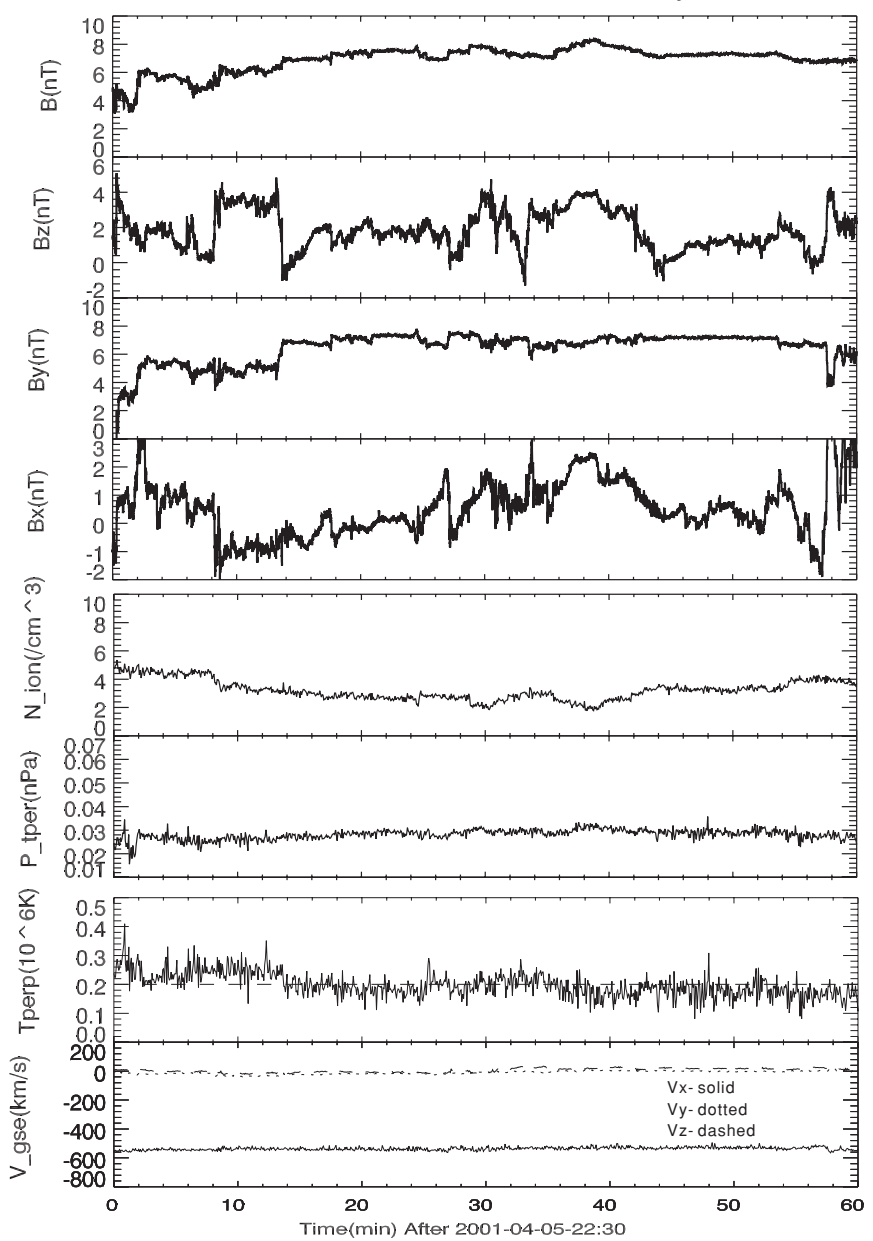

Figure 1. Overview of the measurements from Cluster $\mathrm{C} 1$ satellite between 22:30 and 23:30 UT on 2001 April 5. The first four panels show the magnetic field magnitude and the $x, y, z$ components in Geocentric Solar Ecliptic (GSE) coordinates. The subsequent panels give the ion number density, magnetic pressure plus ion perpendicular pressure, ion temperature perpendicular to the magnetic field, and the velocity components in GSE coordinates.

and $\rho_{i}=v_{i} / \Omega_{c i}$, in which the observed (in the satellite frame) frequency $\omega=2 \pi f$ is mainly due to the Doppler shift, and $\Omega_{c i}$ is the ion cyclotron frequency and $v_{i}$ is its thermal speed. So in our case, the calculated $k \rho_{i}$ value is proportional to this frequency $f$. Besides, it should be mentioned that the PSD of the number density fluctuation we calculated is normalized, which refers to the fluctuations expressed as $\delta N_{\mathrm{e}}=\left(N_{\mathrm{e}}-N_{\mathrm{e}, 0}\right) / N_{\mathrm{e}, 0}$, in which $N_{\mathrm{e}, 0}$ is the averaged value of the electron number density in the studied time period.

\subsection{Observation}

In the time period we chose, we calculated the Alfvén speed and the acoustic wave speed as $V_{A}=B / \sqrt{4 \pi \rho}$ and $C_{s}=\sqrt{\gamma Z k T_{\mathrm{e}} / m_{i}}$, in which $\rho$ is the ion density, $\gamma$ is the adiabatic index set as $5 / 3, Z$ is the ion charge number, $T_{\mathrm{e}}$ is the electron temperature, and $m_{i}$ is the ion mass. Since we find $V_{A}$ is similar to $C_{S}$ (about $90 \mathrm{~km} \mathrm{~s}^{-1}$ ), the solar wind in our case is a compressible fluid. We investigate the relation of $N_{e}$ and $B$ from two aspects: one is their fluctuation spectra and the other is their correlation coefficients.

In Figure 3, we plot the electron density spectrum of the whole $1 \mathrm{hr}$ period from 22:30 to 23:30 UT. It can be identified that the slope of the spectrum is around $-5 / 3$, which is coincident with the results of Marsch \& Tu (1990) in the lowfrequency range. Our work revealed the trend of spectrum to higher frequency. Moreover, the spectrum of the magneticfield-magnitude fluctuations is calculated in the same way as the electron density spectrum. The electron density spectrum has a slope of -1.70 below $k \rho_{i}=0.1$, and is close to the Kolmogorov's $-5 / 3$ power law. In the range of $0.1<k \rho_{i}<1$, the electron density spectrum experiences a relative flattening with a slope of -1.27 , similar to that found by Celnikier et al. (1983, 1987), while the magnetic field spectrum does not show obvious flattening. The uncertainty interval of the spectrum with a 95\% confidence level is shown in Figure 3. It is quite obvious that the fluctuation spectra of $N_{\mathrm{e}}$ and $B$ are extremely similar, which indicates the fluctuation amplitudes of $N_{\mathrm{e}}$ and $B$ change similarly over the various spatial scales. The independence of the amplitude ratio between $N_{\mathrm{e}}$ and $B$ on the value of $k \rho_{i}$ implies that the fluctuations correspond to a set of structures convected by the solar wind flow.

In Figure 4, we study the correlation between the time variations of the electron number density and magnetic field strength. In detail, we first calculate the wavelet cross coherence of $N_{\mathrm{e}}$ and $B$, using the wavelet transformation package from Grinsted et al. (2004), and then compare the time variation of $N_{\mathrm{e}}$ and $B$. Figure 4(a) shows the wavelet cross-coherence spectrum between $N_{\mathrm{e}}$ and $B$ with a period ranging from 10 to $1000 \mathrm{~s}$ and a time interval between 22:30 and 23:30 UT. The left panel shows the spectrum of the coherence coefficient, with its color bar ranging from 0 to 1 as blue to red, and the right panel is the spectrum of the absolute coherence phase angle, with its color bar ranging from 0 to $\pi$ as blue to red. The red-color region inside the black contour lines in the left panel indicates where the cross-coherence coefficient is larger than 0.8 . For easy comparison, these contours are repeated in the right panel. The red-color region in the right panel indicates where the cross-coherence absolute phase angle is close to $\pi$ (i.e., $180^{\circ}$ ). By simultaneous inspection of both panels, we can clearly identify that the red regions limited by the contours have a phase angle of $\pi$ or $-\pi$, which means that here the electron density and magnetic field strength are strongly anti-correlated. This negative correlation extends for large scales over the whole time period considered in Figure 4(a), but it becomes more intermittent and its duration shorter with decreasing time period (from the bottom to top of the panel), thus indicating an increasing number of PBSs as their timescales are reduced.

In addition, we cut out the spectrum with a period of less than $10 \mathrm{~s}$ to meet the requirement of $95 \%$ confidence level for correlation coefficient. Since we have 5 data points per second, we will have 50 data points in $10 \mathrm{~s}$. For the 50 data points, the lowest value of the correlation coefficient to have a $95 \%$ confidence level is 0.27 . However, many of the coefficients in the period lower than $10 \mathrm{~s}$ are less than 0.27 . Thus, we suppose it makes no sense to show the coherence spectrum for times shorter than $10 \mathrm{~s}$.

Figure 4(b) shows the time variation of $N_{\mathrm{e}}$ and $B$ within the regions of the contours in Figure 4(a). The corresponding correlation coefficient is marked in each panel. We list the time series of $N_{\mathrm{e}}$ and $B$ with different anti-correlation timescales, from $1 \mathrm{hr}, 30$ minutes, 10 minutes, 3 minutes to $20 \mathrm{~s}$. In the timescale of $1 \mathrm{hr}$, we find a clear anti-correlation between the electron density and the magnetic field strength, with a correlation coefficient of -0.78 . For the timescale of 30 minutes, between 23:00 and 23:30 UT, the time variations of $N_{\mathrm{e}}$ and $B$ are also anti-correlated with a coefficient of -0.90 . Next, 


\section{Electron Density Calibration}
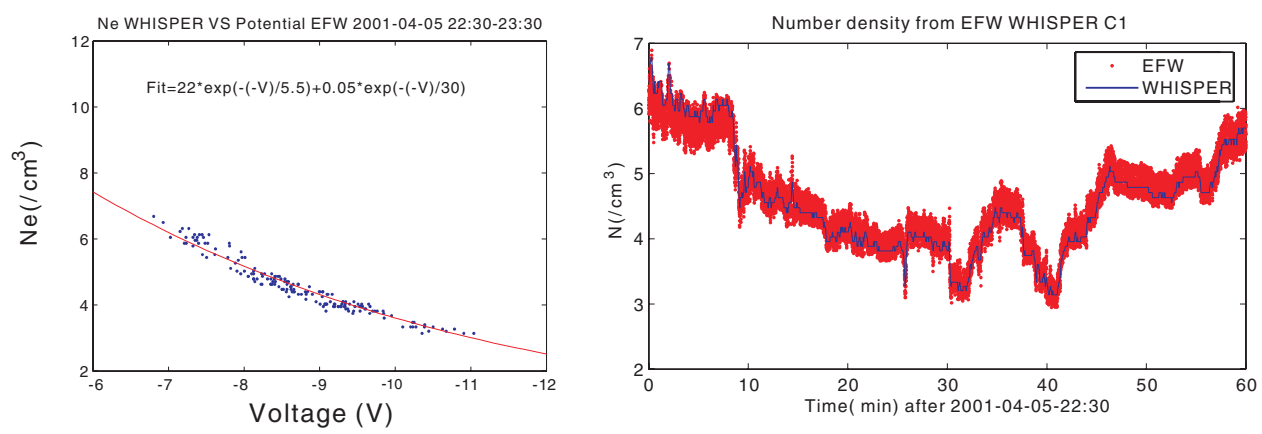

Satellite Spin Effect Filtering
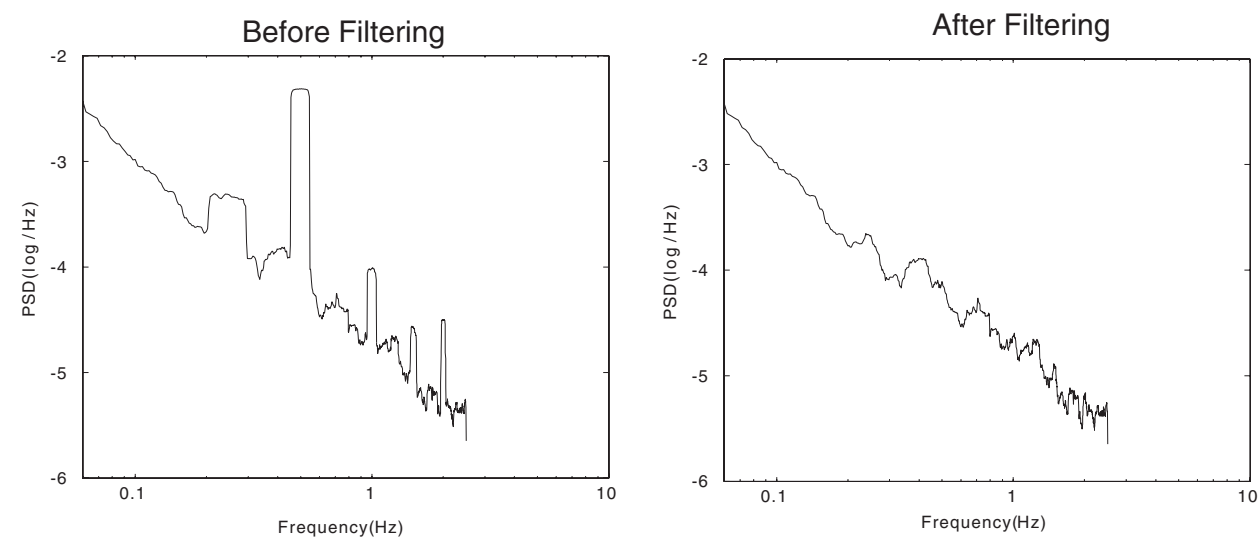

2001-04-05-22:35-22:42 C1

Figure 2. Upper left: the relation between electron number density measured by WHISPER and the spacecraft potential measured by EFW. Upper right: electron number density calculated from the spacecraft potential and the electron number density measured by WHISPER. Lower left: electron density spectrum before notch filtering. Lower right: spectrum after notch filtering.

(A color version of this figure is available in the online journal.)

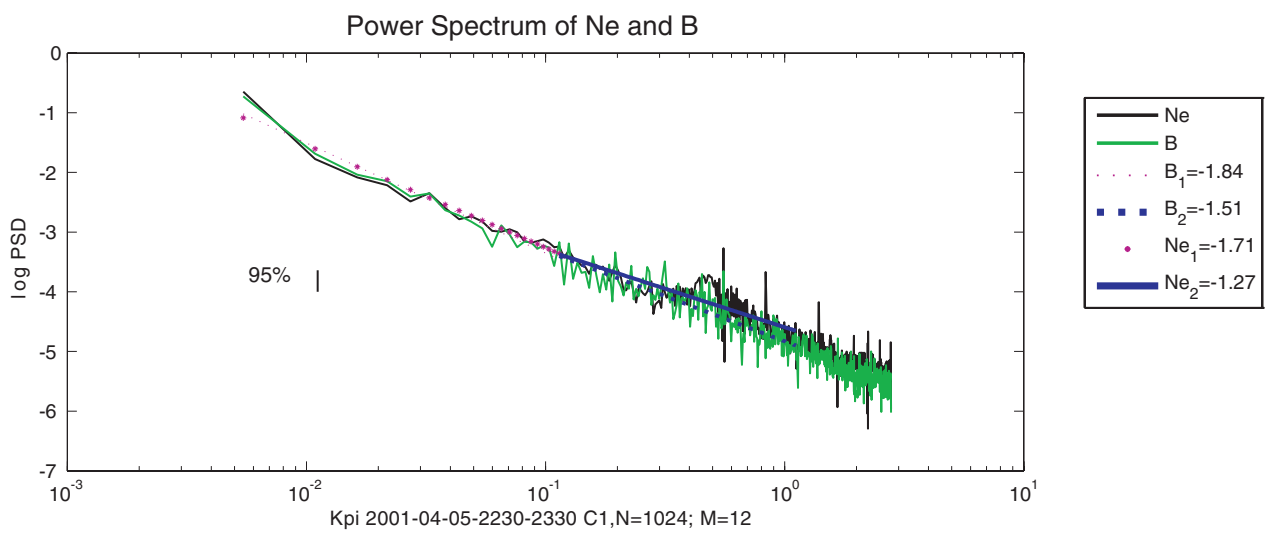

Figure 3. Relative fluctuation spectra of both the electron number density (black solid) and the magnetic field magnitude (green solid). The $x$-axis is $k \rho_{i}$ and the $y$-axis is the logarithmical value of the normalized power spectrum. The spectra are fitted by the least-mean-square method, and the slopes of the fitting results are given in the labels, which are close to $-5 / 3$.

(A color version of this figure is available in the online journal.)

we zoom in to a smaller timescale of 10 minutes. In the time spans 22:50-23:00 UT and 23:00-23:10 UT we find the anticorrelation to exist continuously, with the coefficients being -0.81 and -0.90 , respectively, coincident with the spectrum in Figure 4(a). Even down to a 3 minute timescale, between 23:05 and 23:08 UT, we still find the nice anti-correlation with a coefficient of -0.99 . It should be mentioned that in these above time periods, there were some data gaps caused by measurement failures. We deleted these data gaps and combined the rest of the data points to calculate the correlation coefficients. Furthermore, for the $20 \mathrm{~s}$ scale, between 23:05:30 and 23:05:50 UT, without any data gap, $N_{\mathrm{e}}$ and $B$ are still negatively correlated with a coefficient of -0.96 . In this $20 \mathrm{~s}$ period, the anti-correlation between $N_{\mathrm{e}}$ and $B$ could be identified even on the $1 \mathrm{~s}$ scale. This high correlation coefficient means that the possible pressure balance in this time period was mainly produced by the fluctuations of $N_{\mathrm{e}}$ and $B$. Kellogg \& Horbury (2005) found in many discrete cases that $N_{\mathrm{e}}$ fluctuations in 

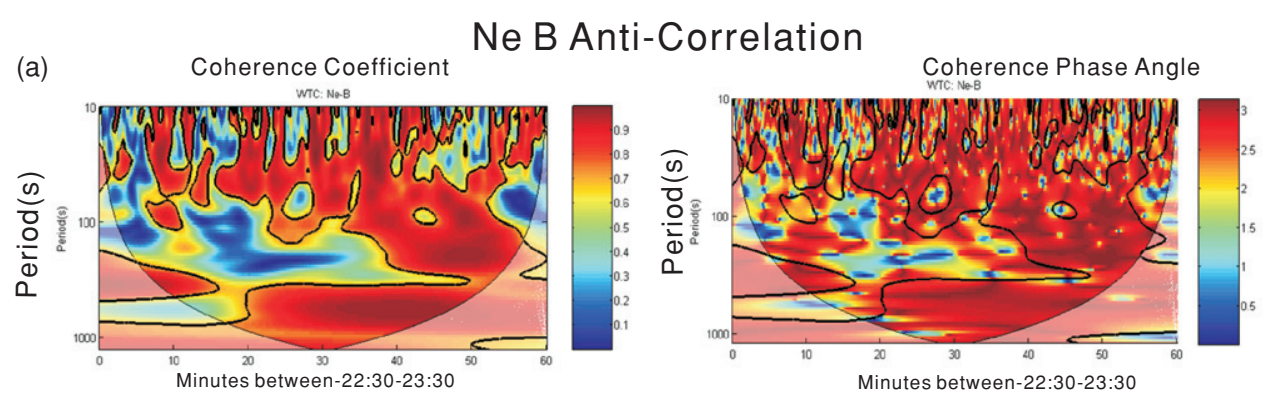

Time Series of $\mathrm{Ne}$ and $\mathrm{B}$

(b)
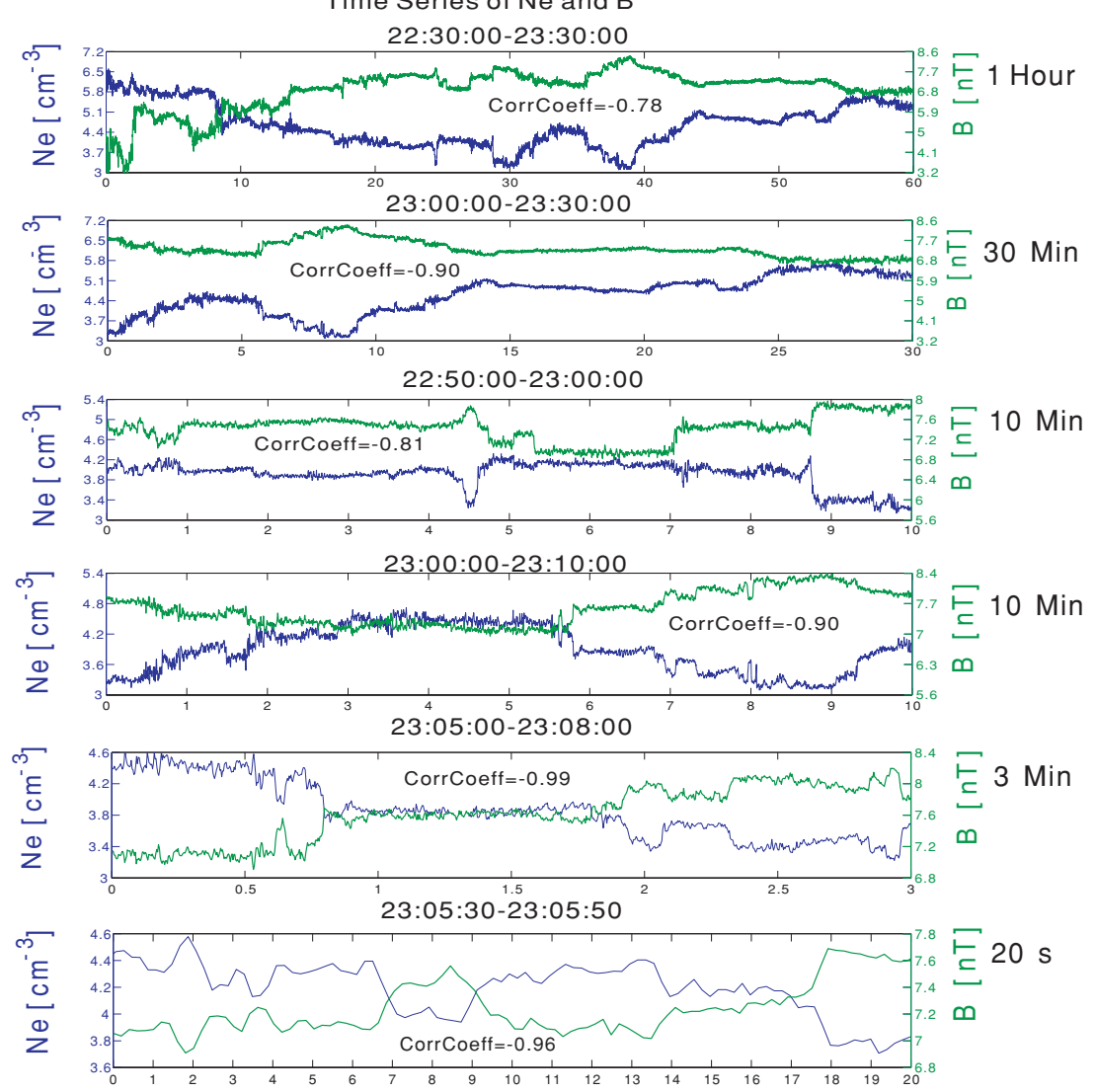

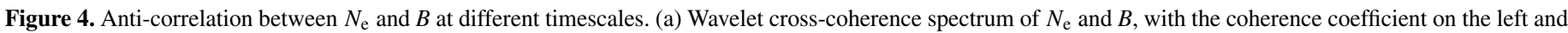

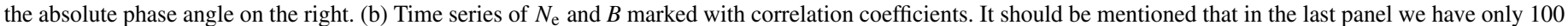

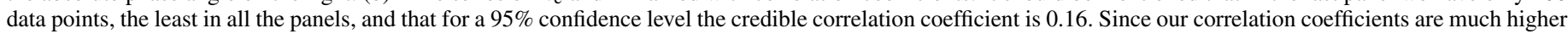
than this critical value, the anti-correlation between $N_{\mathrm{e}}$ and $B$ is reliable.

(A color version of this figure is available in the online journal.)

$0.5 \mathrm{~s}$ scale are accompanied by opposite fluctuations of $B$ in the solar wind period they studied, and they supposed that might be evidence of PBSs. However, they did not analyze the multiscale anti-correlation over the whole solar wind period. We not only confirm the trend they found, but also observe the anticorrelation between $N_{\mathrm{e}}$ and $B$ from second, minute to hour's scale in the whole period we study. Another thing we need to mention is that due to the instrument sampling difference, there is a $0.06 \mathrm{~s}$ time lag between $N_{\mathrm{e}}$ and $B$.

Based on Figures 4(a) and (b), we find anti-correlation between $N_{\mathrm{e}}$ and $B$ with different timescales from 10 to $10^{3} \mathrm{~s}$. According to the anti-correlation between $N_{\mathrm{e}}$ and $B$ and the incomplete total pressure shown in Figure 1, we suppose the solar wind period we study is composed of possible PBSs over multi scales. It probably means that the PBSs can also exist on a small timescale and not only on large timescales in solar wind turbulence, and the small PBSs may be embedded in the large ones. These possible small-scale PBSs may be called mini PBSs in the solar wind at $1 \mathrm{AU}$.

\section{CONCLUSIONS}

We applied the method from Pedersen et al. (2001, 2008) and Kellogg \& Horbury (2005) to derive the time series of the electron number density and its power spectrum, which touches the dissipation range at scales smaller than ion gyro scale. For the first time, we find the anti-correlation between the fluctuations of $N_{\mathrm{e}}$ and $B$ from their power spectra and their wavelet crosscoherence spectra over a large range of scales from $1 \mathrm{hr}$ down to $10 \mathrm{~s}$ during the $1 \mathrm{hr}$ interval we study.

We analyzed the relation between the fluctuations of $N_{\mathrm{e}}$ and $B$ from their power spectra and their wavelet cross-coherence spectra for timescales ranging from $1 \mathrm{hr}$ down to $10 \mathrm{~s}$.

Our work for the first time simultaneously offers the spectra of the electron density and the magnetic field strength up 
to $2.5 \mathrm{~Hz}$ from the in situ measurements made by Cluster, finding these two spectra almost the same in shape and show a Kolmogorov-like power law. This indicates that the relative fluctuations of $N_{\mathrm{e}}$ and $B$ resemble each other, with a similar fluctuation magnitude at the same frequency. There is no obvious dependence of $\delta N_{\mathrm{e}} / \delta B$ on the frequency in the whole frequency range analyzed, a result which cannot be accounted for by the waves with nonlinear dispersion relation over the wide frequency range. A reasonable explanation seems to be multiscale structures convected by the solar wind. Goldreich \& Sridhar (1995) and Montgomery et al. (1987) indicated that the magnetic pressure fluctuations can directly contribute to the power spectrum of the density fluctuations, and as a result the power spectrum of the density fluctuation would attain the same shape as that of the magnetic field fluctuations.

Besides, the electron spectrum in our case has a flatter middle part ranging from 0.1 to $1 \mathrm{~Hz}$. This phenomenon has been observed by Celnikier et al. (1983) as well. And recent work by Chandran et al. (2009) indicates that this spectrum flattening may be contributed by kinetic Alfvén waves (KAWs). Chandran et al. (2009) showed that due to being affected by KAW the Kolmogorov's spectrum would be mixed with a compressional KAW component having a slope of $1 / 3$. Thus, the middle part of the electron density spectrum is a mixture of spectra with slopes of $-5 / 3$ and $1 / 3$. However, we could not exclude the effect on the spectrum from the spacecraft spin at the same time. Since we have the $-5 / 3$ slope of both the electron density spectrum and the magnetic field strength spectrum, we suppose that the solar wind period we studied is still dominated by a passive cascade in the solar wind turbulence.

Furthermore, we investigated the relation between $N_{\mathrm{e}}$ and $B$ to seek for the possible existence of PBSs in the compressible solar wind at $1 \mathrm{AU}$. We detected possible PBSs at different timescales from the in situ observations, based on the anticorrelation between $N_{\mathrm{e}}$ and $B$ inferred from both the time series and the wavelet cross-coherence spectrum. The crosscoherence spectrum shows an anti-correlation between them on the timescales from 10 to $1000 \mathrm{~s}$, which is in agreement with previous work about the anti-correlation between $N_{\mathrm{e}}$ and $B$ in the 1 hr scale (e.g., Bavassano et al. 2004). Differently from that previous work, we find possible PBSs over large scales from hour down to second in solar wind at 1 AU. Since the ion radius $\rho_{i}$ is about $130 \mathrm{~km}$ and the smallest possible PBSs we observed are about $500 \mathrm{~km}$ in extent, these possible PBSs are in size rather close to the ion scale that limits the MHD range from below.

There may be various explanations for the formation of these small possible PBSs. According to Tu \& Marsch (1995), the large fluid-scale PBSs, whose duration is from hours to days, within the inner heliosphere may originate from the Sun; they were also called "spaghetti-like" (Mariani et al. 1973, 1983; McCracken \& Ness 1966; Neugebauer 1981) substructures. Yet the possible PBSs, detected by our new observations of multiscale anti-correlation between $N_{\mathrm{e}}$ and $B$, seem to be mini (kinetic scale) pressure-balanced flux tubes. Previous observations only yielded larger PBSs, which have a duration longer than $1 \mathrm{hr}$ and may originate from the magnetic network in the solar atmosphere (Thieme et al. 1990), but here we probably observed much smaller or even mini PBSs that have short periods from minutes to seconds. Considering their extremely small sizes, these pressure-balanced flux tubes may have their solar source inside the magnetic granulation elements having a size smaller than $70 \mathrm{~km}$. But, this speculation needs confirmation from future higher-resolution observation of the solar atmosphere, as well as from a study of the radial interplanetary evolution of the mini flux tubes. On the other hand, the small possible PBSs may be formed in situ and be driven by some waves or instabilities, for example, by nonlinear Alfvén waves (Vasquez \& Hollweg 1999), or by mirror instability. Another possible mechanism producing the mini structures could be passive cascading of $N_{\mathrm{e}}$ and $B$, which is forced by the active cascading of the velocity fluctuations in the solar wind turbulence. This could explain the observed similarity of the two compressive fluctuation spectra in Figure 3 as well, as they are driven by the same mechanism.

The data in this work are from the Cluster Active Archive. S.Y. thanks E. Lucek and the FGM team. The work done by C.-Y. T. and S.Y. is supported by the National Natural Science Foundation of China under contracts 40874090, 40931055, and 40890162.

\section{REFERENCES}

Alexandrova, O., Carbone, V., Veltri, P., \& Sorriso-Valvo, L. 2008, ApJ, 674, 1153

Alexandrova, O., Saur, J., Lacombe, C., Mangeney, A., Mitchell, J., Schwartz, S. J., \& Robert, P. 2009, Phys. Rev. Lett., 103, 165003

Bavassano, B., Pietropaolo, E., \& Bruno, R. 2004, Ann. Geophys., 22, 689

Burlaga, L. F., \& Ogilvie, K. W. 1970, Sol. Phys., 15, 61

Celnikier, L. M., Harvey, C. C., Jegou, R., Kemp, M., \& Moricet, P. 1983, A\&A, 126,293

Celnikier, L. M., Muschietti, L., \& Goldman, M. V. 1987, A\&A, 181, 138

Chandran, B. D. G., Quataert, E., Howes, G. G., Xia, Q., \& Pongkitiwanichakul, P. 2009, ApJ, 707, 1668

Dastgeer, S., \& Zank, G. P. 2004, ApJ, 602, L29

Eastwood, J. P., Phan, T. D., Bale, S. D., \& Tjulin, A. 2009, Phys. Rev. Lett., 102, 035001

Goldreich, P., \& Sridhar, S. 1995, ApJ, 438, 763

Goldstein, B., \& Siscoe, G. L. 1972, in Solar Wind, ed. C. P. Sonett, P. J. Coleman, \& J. M. Wilcox (NASA Special Publication 308; Washington, DC: NASA), 506

Grinsted, A., Moore, J. C., \& Jevrejeva, S. 2004, Nonlinear Process. Geophys., 11,561

Higdon, J. C. 1984, ApJ, 285, 109

Kellogg, P. J., \& Horbury, T. S. 2005, Ann. Geophys., 23, 3765

Labelle, J., \& Kintner, P. M. 1989, Rev. Geophys., 27, 495

Mariani, F., Bavassano, B., \& Villante, U. 1983, Sol. Phys., 83, 349

Mariani, F., Bavassano, B., Villante, U., \& Ness, N. F. 1973, J. Geophys. Res., 78,8011

Marsch, E., \& Tu, C.-Y. 1990, J. Geophys. Res., 95, 11945

Marsch, E., \& Tu, C. Y. 1993, Ann. Geophys., 11, 659

McComas, D. J., Gosling, J. T., Hammond, C. M., Phillips, J. L., Neugebauer, M., Balogh, A., \& Forsyth, R. J. 1995, J. Geophys. Res., 100, 19893

McCracken, K. G., \& Ness, N. F. 1966, J. Geophys. Res., 71, 3315

Montgomery, D., Brown, M. R., \& Matthaeus, W. H. 1987, J. Geophys. Res., 92,282

Neugebauer, M. 1981, Fundam. Cosm. Phys., 7, 131

Pedersen, A., et al. 2001, Ann. Geophys., 19, 1483

Pedersen, A., et al. 2008, J. Geophys. Res., 113, A07S33

Percival, D. B., \& Walden, A. T. 1993, Spectral Analysis for Physical Applications: Multitaper and Conventional Univariate Techniques (Cambridge: Cambridge Univ. Press), 331

Reisenfeld, D. B., McComas, D. J., \& Steinberg, J. T. 1999, Geophys. Res. Lett., 26,1805

Rème, H., et al. 2001, Ann. Geophys., 19, 1303

Schekochihin, A. A., Cowley, S. C., Dorland, W., Harmett, G. W., Howes, G. G., Quataert, E., \& Tatsuno, T. 2009, ApJS, 182, 310

Thieme, K. M., Marsch, E., \& Schwenn, R. 1990, Ann. Geophys., 8, 713

Tjulin, A., Sahraoui, F., André, M., \& Cornilleau-Wehrlin, N. 2005, J. Geophys. Res., 110, A11224

Tu, C.-Y., \& Marsch, E. 1994, J. Geophys. Res., 99, 21481

Tu, C.-Y., \& Marsch, E. 1995, Space Sci. Rev., 73, 1

Vasquez, B. J., \& Hollweg, J. V. 1999, J. Geophys. Res., 104 4681 